\title{
DGRh hat Handlungsempfehlungen aktualisiert
}

Wie wirkt sich eine entzündlich-rheumatische Erkrankung auf das Covid-19-Risiko aus? Wie wirksam ist die Covid-19-Impfung bei Menschen mit Rheuma? Welchen Einfluss haben Rheumamedikamente auf Erkrankungsrisiko und Impferfolg? Während diese Fragen zu Beginn der Pandemie noch nicht sicher beantwortet werden konnten, haben Mediziner und Wissenschaftler mittlerweile eine Fülle von Erfahrungen mit dem Virus gesammelt. Diesem Erkenntnisgewinn trägt die Deutsche Gesellschaft für Rheumatologie e.V. (DGRh) nun mit einer aktualisierten Handlungsempfehlung zu Covid-19 und Rheuma Rechnung, die sich sowohl an rheumatologisch arbeitende Ärzte, als auch, in einer gesonderten Fassung, an die Patienten richtet.

Wichtig ist den DGRh-Experten zunächst eine beruhigende Nachricht: Eine entzündlich-rheumatische Erkrankung per se gilt nicht als Risikofaktor für eine Infektion mit SARS-CoV-2 oder für einen schweren Covid-19-Verlauf. „Natürlich sollten sich Rheumabetroffene ebenso wie alle anderen an die vom Robert Koch-Institut (RKI) empfohlenen Vorsichtsmaßnahmen halten", sagte Prof. Dr. Christof Specker, Vizepräsident der DGRh und Sprecher der Covid-19-Kommission der Fachgesellschaft.

》) Die Krankheitskontrolle hat oberste Priorität, Medikamente nie selbstständig absetzen

„Darüber hinausgehende Maßnahmen sind aber nicht erforderlich." Insbesondere sollten Patientinnen und Patienten nicht aus Angst vor einer Infektion Arzttermine absagen oder auf eigene Faust ihre Medikation ändern. Denn - auch das haben die bisher verfügbaren Studien ergeben - von den in der Rheumatherapie eingesetzten immunmodulierenden oder immunsupprimierenden Medikamenten geht mit wenigen Ausnahmen kein erhöhtes Covid-19-Risiko aus. Eine medikamentös unzureichend kontrollierte, in einem aktiven Schub befindliche Rheumaerkrankung dagegen gilt durchaus als Risikofaktor für einen schweren Covid-19-Verlauf.,Die Krankheitskontrolle hat daher oberste Priorität", so Specker.

Das spiegeln auch die aktuellen DGRh-Empfehlungen wider: Rheuma- betroffene sollten auch und gerade in Corona-Zeiten ihre Medikation unverändert fortsetzen. Erst bei Symptomen einer Covid-19-Erkrankung sollten DMARDs pausiert werden. Dabei sollte eine Kortison-Therapie für die rheumatische Erkrankung bis ca. $10 \mathrm{mg}$ pro Tag auch in diesen Fällen fortgeführt werden, um einen Kortison-Mangel im Rahmen der Infektion zur vermeiden. Bei höheren Dosen als $10 \mathrm{mg}$ täglich muss individuell entschieden werden. Liegt ein positiver PCR-Test vor, aber keine Symptome von COVID-19, kann ein Pausieren bestimmter Rheumamedikamente (ts oder bDMARDs) für wenige Tage erwogen werden, bis man weiß, ob sich überhaupt Krankheitszeichen von COVID-19 entwickeln.

\section{Rheumamedikamente, Impfung und Impferfolg}

Die Experten der DGRh empfehlen grundsätzlich, antirheumatische Medikamente im Vorfeld einer Impfung nicht abzusetzen oder zu pausieren. Auch wenn bestimmte Wirkstoffe die Bildung von Antikörpern abschwächen können, ist damit der Impferfolg in der Regel nicht gefährdet. Eine Ausnahme bildet das Medikament Rituximab, das möglichst in größerem zeitlichem Abstand zur Impfung gegeben werden sollte. Für alle anderen Medikamente gilt, nur nach sorgfältiger Abwägung mit dem behandelnden Rheumatologen zu entscheiden, ob und wann bei einzelnen Substanzen und bei stabiler Krankheitskontrolle eine kurze Medikamentenpause sinnvoll ist, um eine möglichst gute Impfantwort zu erreichen. „Eine gute Krankheitskontrolle ist auch hier wichtiger als das Erreichen einer optimalen Immunantwort auf die Impfung", betont DGRhPräsident Prof. Dr. Andreas Krause aus Berlin.

Den Antikörperspiegel nach der Impfung zu überprüfen kann die DGRh nicht generell empfehlen: „Die Bedeutung des Antikörpertiters für den Immunschutz gerade bei der Infektion mit SARS-CoV-2, welcher durch neutralisierende Antikörper und zytotoxische T-Zellen vermittelt wird, ist unbekannt. Daher ist die Bestimmung der Antikörper für eine Aussage zum Immunschutz nicht geeignet", betont Prof. Dr. Hendrik Schulze-Koops, 2. Vizepräsident der DGRh aus München und stellvertretender Sprecher der Covid19-Kommission.

\section{Keine erhöhten Impf- Nebenwirkungen}

Studien, die sich mit dem Nebenwirkungsprofil der Impfungen speziell bei Rheumapatienten befassen, sind bislang dünn gesät. „Die Erhebungen, die es gibt, deuten jedoch nicht auf eine besondere Unverträglichkeit hin", sagt Krause. Auch bei weltweit nunmehr 2,4 Milliarden verabreichten Impfungen gebe es keinen Anhaltspunkt dafür, dass Rheumapatienten mit mehr oder schwereren Nebenwirkungen zu rechnen hätten. Generell können alle vier in der EU zugelassenen Covid19-Impfstoffe auch für diese Patientengruppe als sicher und wirksam gelten. Allerdings raten die DGRh-Experten dazu, bei Menschen, deren rheumati- 


\section{Springer Medizin}

sche Grunderkrankung mit einer Störung der Blutgerinnung verbunden ist - etwa bei einem AntiphospholipidSyndrom oder einer Immunthrombopenie - eher einen mRNA-Impfstoff zu verwenden.

\section{Online-Register liefert Evidenz}

In die aktuellen Handlungsempfehlungen sind die Ergebnisse einer Vielzahl internationaler Studien zum Thema eingeflossen. „Eine wertvolle Quelle ist auch das online-Register Covid19-rheuma.de, das auf Initiative der DGRh bereits im letzten Frühjahr gestartet wurde. Darin sind mittlerweile Covid-19-Krankheitsverläufe von 2900 Rheumapatienten dokumentiert", berichtet Specker, der an der Erstellung der Handlungsempfehlungen federführend beteiligt war. Eine der aktuellen
Empfehlungen lautet daher auch: Deutsche Rheumabetroffene, die positiv auf SARS-CoV-2 getestet werden, sollten in das Online-Register aufgenommen und der Verlauf der Erkrankung sollte dort dokumentiert werden.

\section{Weitere Informationen:}

Specker, C et al: Aktualisierte Handlungsempfehlungen der Deutschen Gesellschaft für Rheumatologie für die Betreuung von Patienten mit entzündlichrheumatischen Erkrankungen im Rahmen der SARS-CoV-2/COVID-19-Pandemie einschließlich Empfehlungen zur COVID-19 Impfung: https://bit.ly/3wtQzMs

Patientenversion zu den Handlungsempfehlungen der Deutschen Gesellschaft für Rheumatologie für die Betreuung und Impfung von Patienten mit entzündlichrheumatischen Erkrankungen im Rahmen der Coronavirus-Pandemie: https://bit.ly/ $36 q T s 68$

In Österreich können Daten von Rheumapatienten, auch von jenen, die positiv auf SARS-CoV-2 getestet werden, im BioReg dokumentiert werden: www.bioreg.at

Hinweis des Verlags. Der Verlag bleibt in Hinblick auf geografische Zuordnungen und Gebietsbezeichnungen in veröf-

fentlichten Karten und Institutsadressen neutral.

rheuma plus $2021 \cdot 20: 152-153$

https://doi.org/10.1007/s12688-02100444-9

Online publiziert: 19. Juli 2021

(c) Springer-Verlag GmbH Austria, ein Teil von Springer Nature 2021
Quelle: Presseaussendung der Deutschen Gesellschaft für Rheumatologie e.V. (DGRh)

Hier steht eine Anzeige. 